\title{
Studentenbuch, Praxisbuch und Nachschlagewerk der Radiologie
}

Hatem Alkadhi

Korrespondenz: PD Dr. med. Hatem Alkadhi UniversitätsSpital Zürich Institut für Diagnostische und Interventionelle Radiologie Rämistrasse 100 CH-8091 Zürich

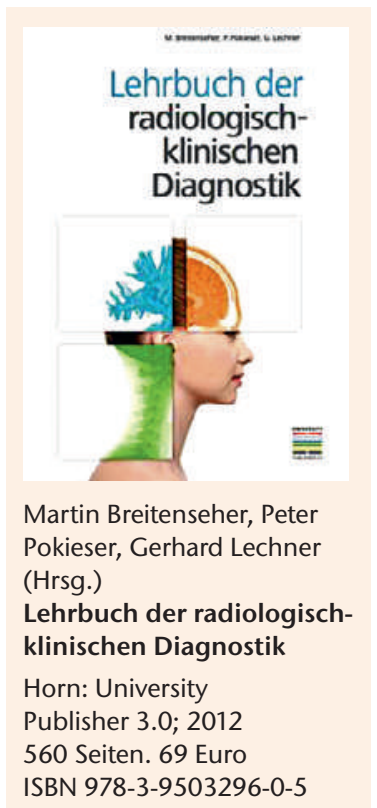

Die Professoren Breitenseher, Pokieser, Lechner, allesamt Radiologen aus Wien, haben im Januar 2012 eine empfehlenswerte Neuauflage des 2003 erstmals erschienenen Lehrbuchs der radiologisch-klinischen Diagnostik herausgegeben. Auf 560 Seiten liefern die insgesamt 52 Kapitel-Autoren eine Mischung aus physikalisch-technischen sowie klinischen Aspekten der Radiologie.

Die Einteilung

der kurzen und prägnanten Kapitel reflektiert auf treffliche Weise die aktuellen Entwicklungen im Fach der Radiologie. So finden sich im ersten Teil des Buches gerätebasierte Kapitel mit den technischen Grundlagen der digitalen Radiographie, Ultrasonographie, Computertomographie, Magnetresonanztomographie und der Nuklearmedizin sowie der in der Radiologie üblichen Kontrastmittel. Daran schliessen sich krankheitsbasierte Kapitel an, die u. a. die Notfallradiologie mit der Traumatologie von Knochen, Weichteilen und inneren Organen oder die Bildgebung bei Aids zusammenfassen. Im nächsten Teil folgen organbasierte Kapitel z. B. über die Bildgebung von Krankheiten der Leber und Milz. Schliesslich finden sich in diesem umfassenden Buch auch Kapitel über Rechtsfragen und über die Evidenz in der Radiologie.

Wie man sich gut vorstellen kann, ist es sehr schwierig, derart viele Themengebiete in nur einem einzigen Buch zu vereinen. Dies ist den Autoren mit einer Vielzahl anschaulicher und instruktiver Abbildungen, die das Lesen und Verstehen der verschiedenen bildgebenden Aspekte der verschiedenen Geräte, Krankheiten und Organe erleichtern, auf treffliche Weise gelungen. Zusätzlich helfen zahlreiche Skizzen, wesentliche radiologische Befunde und anatomische Zusammenhänge verständlich zusammenzufassen. Ein weiterer Pluspunkt dieses Buches ist das Layout. In den einzelnen Kapiteln tauchen immer wieder blau unterlegte Kästchen auf, die durch die Farbkennzeichnung auf jeweils praxisrelevante Tipps und Tricks hinweisen.

Das Buch ist als «Studentenbuch», «Praxisbuch» und «Nachschlagewerk» für die radiologisch-klinische Diagnostik konzipiert und kann aufgrund der Vielzahl und der Breite der darin behandelten Themen ein Begleiter für angehende Ärzte im Studium bis hin zur klinischen Tätigkeit sein. Darüber hinaus kann es auch für MTRA/RTs als Nachschlagewerk in der Ausbildung wie auch in der täglichen Arbeit dienen. Für vertieftes Lesen im Rahmen der Weiterbildung zum Radiologen kann dann auf spezifischere Bücher und Literatur übergegangen werden. 